\title{
Non-invasive assessment of left ventricular response to Valsalva manoeuvre in normal and diabetic subjects using praecordial accelerocardiography
}

\author{
LESLIE HUME, DAVID J. EWING, IAN W. CAMPBELL, \\ STUART R. REUBEN, AND BASIL F. CLARKE
} From the University Department of Medicine and the Diabetic and Dietetic Department,
The Royal Infirmary, Edinburgh

SUMMARY The left ventricular response to the Valsalva manoeuvre was studied in 5 normal subjects (group 1), 6 diabetics without autonomic neuropathy (group 2), and 5 diabetics with autonomic neuropathy (group 3), using the maximum amplitude of the praecordial accelerocardiogram (DE) as a noninvasive index of left ventricular performance.

During the Valsalva manoeuvre DE decreased in all 3 groups. In groups 1 and 2, DE increased significantly above the control value after release of the manoeuvre (DE overshoot) but this did not occur in group 3. It is suggested that the overshoot of $\mathrm{DE}$ in groups 1 and 2 reflects an increase in left ventricular contractility after release of the Valsalva manoeuvre and the absence of an overshoot in $\mathrm{DE}$ in the patients with autonomic neuropathy is the result of loss of cardiac adrenergic innervation.

The ability to detect an abnormal cardiovascular response to the Valsalva manoeuvre using the non-invasive technique of praecordial accelerocardiography may be of practical value in the assessment of left ventricular function.

Praecordial accelerocardiography is a useful noninvasive technique for detecting changes in peak acceleration of aortic blood flow, which itself is a sensitive measure of left ventricular performance (Reuben and Littler, 1973).

There is normally an increase in left ventricular contractility after release of the Valsalva manoeuvre (Mason et al., 1970; Brooker et al., 1974) but the haemodynamic response to the manoeuvre is often impaired in patients with heart disease (Elisberg, 1963). An abnormal cardiovascular response to the Valsalva manoeuvre also occurs commonly in patients with diabetic autonomic neuropathy (Sharpey-Schafer and Taylor, 1960; Lloyd-Mostyn and Watkins, 1975).

The present study was designed to see whether the changes in left ventricular contractility during and after the Valsalva manoeuvre could be detected non-invasively using praecordial accelerocardiography in normal subjects and to compare this with the left ventricular response in diabetic patients with known autonomic neuropathy.

Received for publication 20 March 1978

\section{Subjects and methods}

The patients studied were 5 normal male subjects (group 1), 6 male diabetics with no clinical features of autonomic neuropathy or other diabetic complications and with a normal Valsalva ratio (group 2), and 5 male diabetics with clinical features of autonomic neuropathy (postural hypotension in 4, abnormal sweating in 4, diarrhoea in 4, gastric fullness in 2, hypoglycaemic unawareness in 2) (group 3); all of the latter had an abnormal Valsalva ratio and sustained handgrip response (Ewing et al., 1973). In group 1 the mean age was 47 (range 35 to 60) years, in group 2 it was 45 (range 21 to 54) years, and in group 3 it was 43 (range 28 to 65) years. The mean duration of diabetes in group 2 was 19 (range 7 to 36) years and in group 3 it was 20 (11 to 29) years. All but 2 of the diabetics were insulin-dependent. None of the normal or diabetic subjects had clinical, electrocardiographic, or radiological evidence of cardiovascular disease.

The praecordial accelerocardiogram transducer, which has been described in detail previously (Reuben and Littler, 1973; Hume et al., 1975), was 
attached by an adhesive disc to the chest wall overlying the fifth rib at a point medial to the apex beat. A simultaneous electrocardiogram was recorded with the accelerocardiogram on the second channel of an ultraviolet recorder.

The subjects performed a standardised Valsalva manoeuvre while lying supine by blowing into a mouthpiece attached to a manometer and maintaining a pressure of $40 \mathrm{mmHg}$ for 15 seconds (Ewing et al., 1973). The electrocardiogram and accelerocardiogram were recorded continuously for 15 seconds before, during, and for 20 cardiac cycles after release of the manoeuvre.

In each subject the $R R$ interval and maximum amplitude of the accelerocardiogram (DE deflection according to labelling of Luisada, 1962, which occurs at the onset of ejection) were averaged over 10 consecutive cardiac cycles immediately preceding the onset of the Valsalva manoeuvre in order to obtain control values. The RR interval and DE were also averaged over the last 5 beats before release of the Valsalva strain. After release the RR interval and $\mathrm{DE}$ were measured individually for the next 20 beats. The $R R$ interval and DE during and after the Valsalva manoeuvre were expressed as a percentage of the control value. No attempt was made to calibrate the tracings in absolute units of acceleration because individual variations in chest wall thickness and transducer coupling mean that the absolute value of $\mathrm{DE}$ has no physiological significance.
The significance of the changes in DE during the Valsalva manoeuvre within each group was calculated by Student's paired t test, using the original measurements of DE. When comparing the percentage changes in DE during the Valsalva manoeuvre between the different groups, one-way analysis of variance was used.

\section{Results}

The Table shows the mean $R R$ interval and $D E$ in each group during the Valsalva manoeuvre and for 20 beats after release, expressed as a percentage of the control value. Fig. 1 is an example of a normal response and Fig. 2 shows the time relation of the changes in $R R$ interval and $D E$ in the different groups. As can be seen from Fig. 2, the pattern of response in terms of $R R$ interval and $D E$ was similar in groups 1 and 2 .

RR INTERVAL (Table; Fig. 2)

In all 3 groups the mean $R R$ interval decreased during the Valsalva manoeuvre. Immediately on release there was a further small decrease lasting 4 to 5 beats, after which the RR interval increased to reach the control value by beat 7 to 11 in groups 1 and 2 . Whereas the $R R$ interval increased above the control value in groups 1 and 2 , reaching a maximum between beats 13 and 20, in group 3 no increase in $R R$ interval above the control value was observed after release of the Valsalva manoeuvre.

Table Group mean changes in $R R$ interval and $D E$ during Valsalva manoeuvre (expressed as percentage of control mean value)

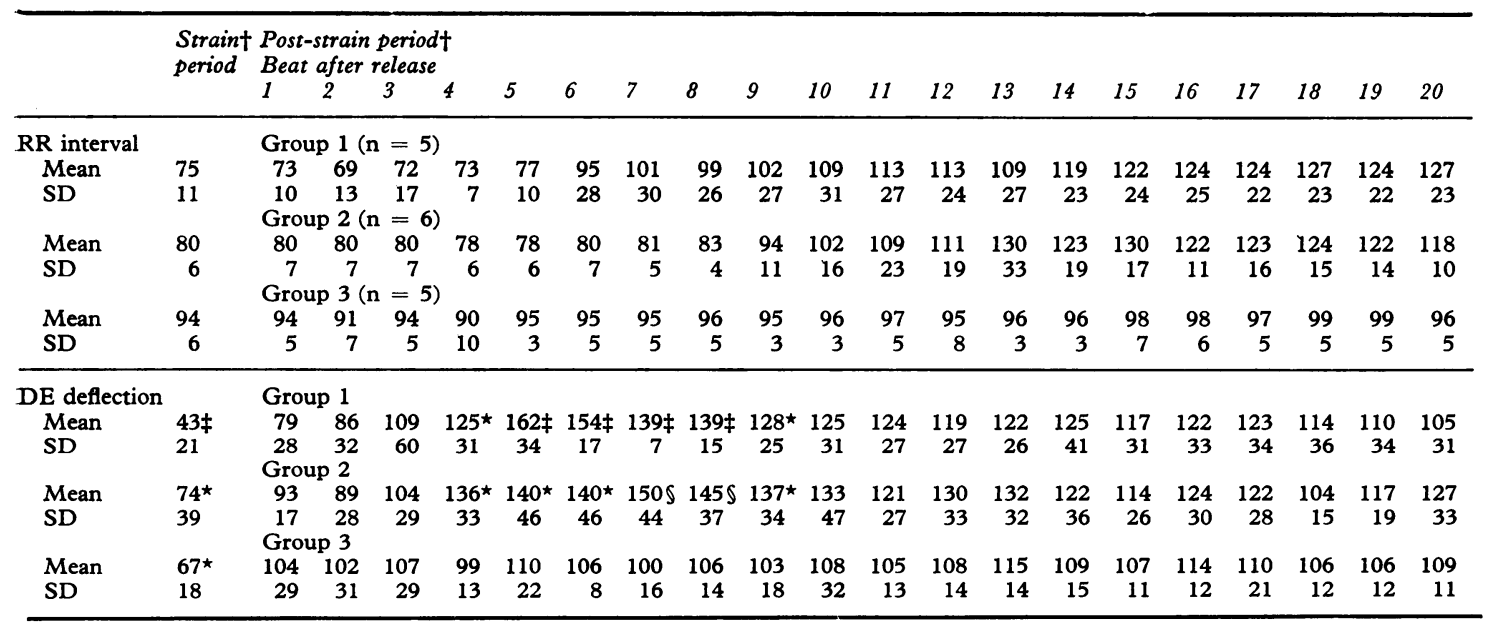

tValues of RR and DE expressed as a percentage of the average value for 10 consecutive cardiac cycles immediately preceding the Valsalva strain.

During strain period RR and DE are expressed as average of values for 5 consecutive cardiac cycles immediately before release of Valsalva. Significance of change from control value, $\star P<0.05 ; § P<0.01 ; \ddagger P<0.001$. 


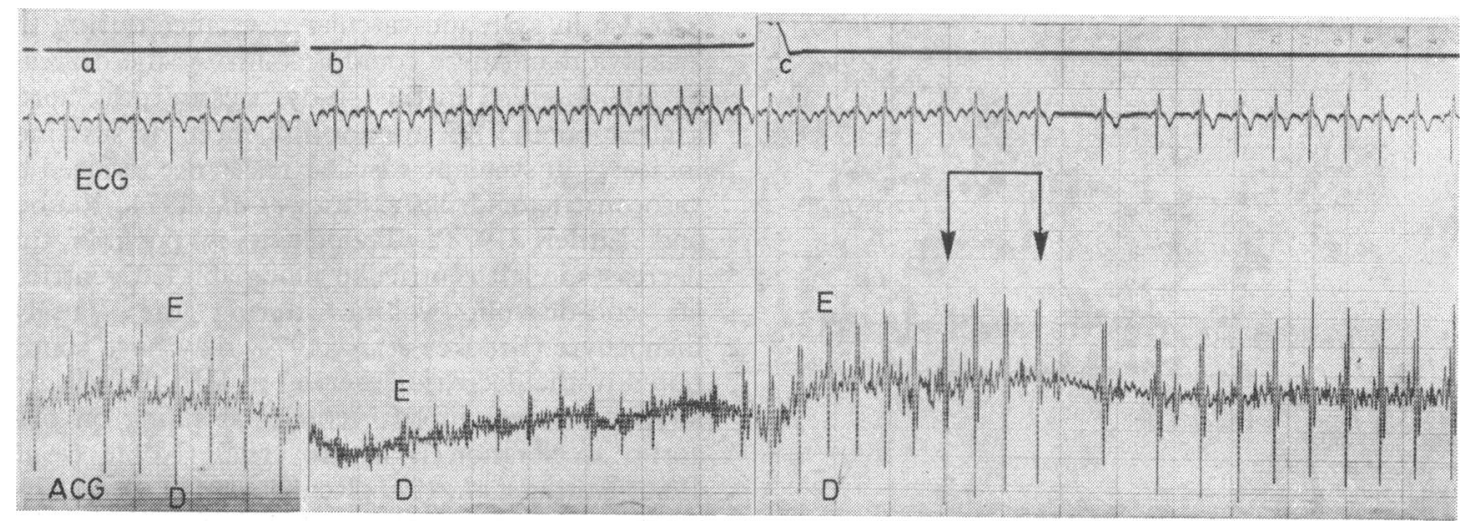

Fig. 1 Example of a normal response to the Valsalva manoeuvre. (a) Before Valsalva strain. (b) During Valsalva strain. (c) After release of Valsalva strain. ECG, electrocardiogram; ACG, praecordial accelerocardiogram; $D E$, maximum systolic deflection of accelerocardiogram. Note decrease in DE during Valsalva manoeuvre (b) and overshoot after release (c between arrows) preceding bradycardia.

MAXIMUM AMPLITUDE OF AGCELEROCARDIOGRAM (DE) (Table ; Fig. 2)

In all 3 groups the mean value of $\mathrm{DE}$ decreased significantly during the Valsalva manoeuvre (group 1 $\mathbf{P}<0.001$; group $2 \mathrm{P}<0.05$; group $3 \mathbf{P}<0.05$ ). After release, DE increased to reach the control value within 3 beats in all 3 groups. Thereafter the mean value for $\mathrm{DE}$ increased significantly above the control value from beats 4 to 9 inclusive in groups 1 and 2, before decreasing again. All 11 subjects in groups 1 and 2 showed this characteristic 'overshoot' in DE. In group 3 no significant increase over the control value of $\mathrm{DE}$ occurred at any time after release of the Valsalva manoeuvre in the group as a whole, though 1 subject in this group did show an 'overshoot' in DE after release of the manoeuvre (that is a value greater than control mean +2 standard deviations of the mean).

When the 3 groups were compared using analysis of variance no significant difference was found between them in the magnitude of the decrease in DE during the Valsalva manoeuvre $(P>0.05)$. After release $\mathrm{DE}$ was significantly greater in groups 1 and 2 than in group 3 from beats 4 to 8 inclusive $(P<0.05$ in each case).

\section{Discussion}

The beat-to-beat changes in $R R$ interval during and after the Valsalva manoeuvre in the normal subjects and the diabetics without autonomic neuropathy in this study are similar to those described previously in normal subjects (Flessas et al., 1970). The response in the diabetics with autonomic neuropathy is also in agreement with previous observa- tions in similar patients (Lloyd-Mostyn and Watkins, 1975).

In addition, the present study shows that the maximum amplitude of the praecordial accelerocardiogram (DE) consistently decreased during the Valsalva manoeuvre and increased significantly above the control value after release (that is 'overshoot') in the normal subjects and the diabetics without autonomic neuropathy. In the diabetics with autonomic neuropathy there was a similar decrease in DE during the Valsalva manoeuvre but no significant increase above the control value occurred after release.

Changes in DE have been shown to correlate closely with changes in the peak acceleration of blood flow in the ascending aorta in dogs during a wide range of manoeuvres (Reuben and Littler, 1973). Peak acceleration of aortic flow, in turn, is very sensitive to changes in left ventricular contractile state induced in dogs by coronary artery occlusion or the administration of various drugs (Noble et al., 1966; Nutter et al., 1971; Reuben and Littler, 1973). The praecordial accelerocardiogram therefore appears to be a useful non-invasive technique for detecting changes in left ventricular performance (Reuben and Littler, 1973).

The effects of the Valsalva manoeuvre on DE in the normal subjects and the diabetics without autonomic neuropathy in this study are in agreement with previous catheterisation studies in which peak aortic blood flow and peak velocity of aortic flow were measured. These indices of left ventricular pump performance were shown to decrease during the Valsalva manoeuvre and to increase above control values after release (Fox et al., 1966; 

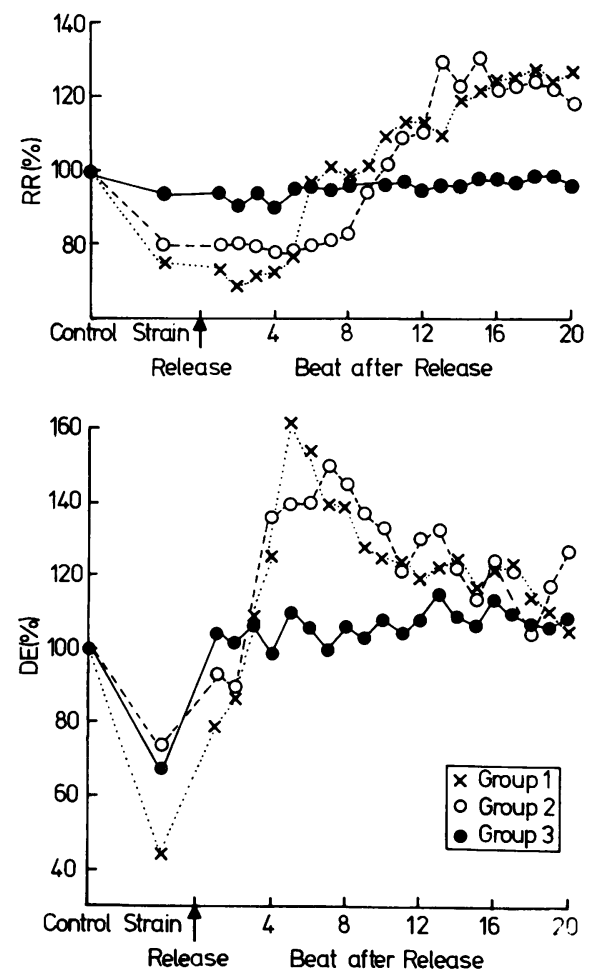

Fig. 2 Group mean changes in RR interval and $D E$ during and after Valsalva manoeuvre. Crosses (group 1), no:mal subjects; open circles (group 2), diabetics without autonomic neuropathy; closed circles (group 3), diabetics with autonomic neuropathy. Note DE overshoot in groups 1 and 2 precedes reflex bradycardia.

Greenfield et al., 1967; Mason et al., 1970). Furthermore, the time course of the 'overshoot' in DE after release of the Valsalva manoeuvre in this study was similar to that observed in peak aortic flow velocity measured in normal subjects at cardiac catheterisation (Greenfield et al., 1967).

Although the decrease in DE during the Valsalva manoeuvre in this study may have been partly the result of a change in the coupling between the heart and chest wall during the forced expiration, the magnitude of the decrease was strikingly similar to that which occurred in peak aortic flow velocity measured at cardiac catheterisation during the Valsalva manoeuvre (Greenfield et al., 1967; Mason et al., 1970). Some observers have attributed the decline in left ventricular pump function during the Valsalva manoeuvre observed in catheterisation studies to the combined effects of increased systemic vascular resistance and reduced left ventricular filling during the manoeuvre (Mason et al., 1970). It seems unlikely, however, that the increase in systemic vascular resistance during the Valsalva manoeuvre could account for the decrease in DE observed in this study because peak aortic accleration and DE are not influenced by sustained increases in systemic vascular resistance induced by vasoconstrictor drugs (Nutter et al., 1971; Reuben and Littler, 1973). Though it is possible that decreases in left ventricular filling and left ventricular end-diastolic volume during the Valsalva manoeuvre (Brooker et al., 1974) may have contributed to the decrease observed in DE, the effect, if any, of changes in left ventricular filling on peak aortic acceleration is controversial (Noble et al., 1966; Nutter et al., 1971; Reuben and Littler, 1973).

After release of the Valsalva manoeuvre left ventricular end-diastolic volume does not increase above the control value (Brooker et al., 1974) and systemic vascular resistance remains increased (Elisberg, 1963). Consequently the 'overshoots' in peak aortic blood flow and peak aortic flow velocity observed in normal subjects at cardiac catheterisation (Fox et al., 1966; Greenfield et al., 1967; Mason et al., 1970) indicate an increase in left ventricular contractile state after release of the Valsalva manoeuvre which was shown in this study using the non-invasive technique of praecordial accelerocardiography.

The absence of an 'overshoot' in DE after release of the Valsalva manoeuvre in the diabetics with autonomic neuropathy suggests that such patients are unable to increase left ventricular contractile state at this time. This may contribute to the impairment or absence of the normal overshoot in blood pressure which is frequently observed in such patients (Sharpey-Schafer and Taylor, 1960) because the presence of a normal blood pressure overshoot depends upon the ability of the left ventricle to increase cardiac output as well as on the patient's ability to increase systemic vascular resistance during and after the Valsalva manoeuvre (Elisberg, 1963). The inability of the diabetic patients with autonomic neuropathy to increase left ventricular contractility after release of the Valsalva manoeuvre is probably caused by loss of cardiac adrenergic innervation. It has been shown that such patients often have a reduced catecholamine response to tilting associated with a subnormal increase in heart rate, suggesting that cardiac adrenergic nerve function is impaired (Christensen, 1972). The contribution which beta-adrenergic stimulation makes to the heart rate changes during the Valsalva manoeuvre in normal subjects is not clear (Elisberg, 1963; Leon et al., 1970), but beta-adrenergic blockade has been shown to abolish the overshoot in blood pressure (Prichard and Gillam, 1966) and may even convert a normal response into the 'square 
wave' type of response which is characteristic of patients with impaired left ventricular function (Vogel and Blount, 1967). These observations suggest that the beta-adrenergic nervous system is involved in the normal inotropic response to the Valsalva manoeuvre.

This study shows that it is possible to detect changes in left ventricular performance during the Valsalva manoeuvre in normal subjects and also to detect an impaired haemodynamic response in diabetics with autonomic neuropathy using the non-invasive technique of praecordial accelerocardiography. Since impairment of the left ventricular response to the Valsalva manoeuvre is helpful in the assessment of cardiac function (Mason et al., 1970; Brooker et al., 1974) this technique may find a clinical application in the evaluation of cardiac patients.

We are grateful to Dr R. J. Prescott for statistical advice.

\section{References}

Brooker, J. Z., Alderman, E. L., and Harrison, D. C. (1974). Alterations in left ventricular volumes induced by Valsalva manoeuvre. British Heart fournal, 36, 713-718.

Christensen, N. J. (1972). Plasma catecholamines in long-term diabetics with and without neuropathy and in hypophysectomized subjects. Fournal of Clinical Investigation, 51, 779-787.

Elisberg, E. I. (1963). Heart rate response to the Valsalva maneuver as a test of circulatory integrity. Fournal of the American Medical Association, 186, 200-205.

Ewing, D. J., Campbell, I. W., Burt, A. A., and Clarke, B. F. (1973). Vascular reflexes in diabetic autonomic neuropathy. Lancet, 2, 1354-1356.

Flessas, A. P., Kumar, S., and Spodick, D. H. (1970). Effects of the Valsalva maneuver on the cardiac systolic intervals: beat-to-beat versus timed analysis. American Heart fournal, 80, 522-531.

Fox, I. J., Crowley, W. P., Jr., Grace, J. B., and Wood, E. H. (1966). Effects of the Valsalva maneuver on blood flow in the thoracic aorta in man. Fournal of Applied Physiology, 21, 1553-1560.
Greenfield, J. C., Jr., Cox, R. L., Hernandez, R. R., Thomas, C., and Schoonmaker, F. W. (1967). Pressure-flow studies in man during the Valsalva maneuver with observations on the mechanical properties of the ascending aorta. Circulation, 35, 653-661.

Hume, L., Irving, J. B., Kitchin, A. H., and Reuben, S. R. (1975). Effects of sustained isometric handgrip on praecordial accelerocardiogram in normal subjects and in patients with heart disease. British Heart fournal, 37, 873-881.

Leon, D. F., Shaver, J. A., and Leonard, J. J. (1970). Reflex heart rate control in man. American Heart fournal, 80, 729-739.

Lloyd-Mostyn, R. H., and Watkins, P. J. (1975). Defective innervation of heart in diabetic autonomic neuropathy. British Medical fournal, 3, 15-17.

Luisada, A. A. (1962). (Ed.) Cardiology, an Encyclopedia of the Cardiovascular System. Vol. 2. Suppl. on Methods, part 3. McGraw-Hill, New York.

Mason, D. T., Gabe, I. T., Mills, C. J., Gault, J. H., Ross, J., Jr., Braunwauld, E., and Shillingford, J. P. (1970). Applications of the catheter-tip electromagnetic velocity probe in the study of the central circulation in man. American fournal of Medicine, 49, 465-471.

Noble, M. I. M., Trenchard, D., and Guz, A. (1966). Left ventricular ejection in conscious dogs. 1 . Measurement and significance of the maximum acceleration of blood from the left ventricle. Circulation Research, 19, 139-147.

Nutter, D. O., Noble, R. J., and Hurst, V. W., III (1971). Peak aortic flow and acceleration as indices of ventricular performance in the dog. Fournal of Laboratory and Clinical Medicine, 77, 307-318.

Prichard, B. N. C., and Gillam, P. M. S. (1966). Propranolol in hypertension. American fournal of Cardiology, 18, 387-393.

Reuben, S. R., and Littler, W. A. (1973). Praecordial accelerometry: an indirect assessment of left ventricular performance. European fournal of Clinical Investigation, 3, 324-330.

Sharpey-Schafer, E. P., and Taylor, P. J. (1960). Absent circulatory reflexes in diabetic neuritis. Lancet, 1, 559-562.

Vogel, J. H. K., and Blount, S. G., Jr. (1967). Modification of cardiovascular responses by propranolol. British Heart fournal, 29, 310-316.

Requests for reprints to Dr L. Hume, Department of Medicine, The Royal Infirmary, Edinburgh EH3 9YW. 